\title{
Dinâmica floral e abortamento de flores em híbridos de canola e mostarda castanha
}

\author{
Rafael Battisti ${ }^{(1)}$, Felipe Gustavo Pilau ${ }^{(2)}$, Luciano Schwerz ${ }^{(3)}$, Lucindo Somavilla ${ }^{(3)}$ e Gilberto Omar Tomm ${ }^{(4)}$
}

\begin{abstract}
(1)Universidade de São Paulo, Escola Superior de Agricultura Luiz de Queiroz, Avenida Pádua Dias, no 11, CEP 13418-900 Piracicaba, SP. E-mail: r.battisti@hotmail.com ${ }^{(2)}$ Universidade Federal de Santa Maria (UFSM), Avenida Roraima, №1.000, Cidade Universitária, Bairro Camobi, CEP 97105-900 Santa Maria, RS. E-mail: fgpilau@smail.ufsm.br (3)UFSM, Campus de Frederico Westphalen, Linha 7 de Setembro, s/no, CEP 98400-000 Frederico Westphalen, RS. E-mail: luagronomia@hotmail.com, lucindosomavilla@hotmail.com ${ }^{(4)}$ Embrapa Trigo, Rodovia BR 285, Km 294, Caixa Postal 451, CEP 99001-970 Passo Fundo, RS. E-mail: tomm@cnpt.embrapa.br
\end{abstract}

Resumo - O objetivo deste trabalho foi avaliar a dinâmica floral e determinar o índice de abortamento de flores de híbridos de canola (Brassica napus) e de mostarda castanha (Brassica juncea), bem como determinar suas relações com as condições meteorológicas do Sul do Brasil. Durante a floração, dez híbridos de canola e dois de mostarda foram avaliados a cada três dias quanto ao número de flores abertas, de síliquas e de flores abortadas. O número acumulado e relativo de flores foi usado para avaliação da dinâmica floral. A relação desses números com a soma térmica acumulada durante a floração foi determinada por meio de modelo logístico. A partir dos coeficientes desse modelo, identificaram-se grupos de genótipos com diferentes taxas de emissão de flores. O abortamento de flores entre híbridos variou de 10,53 a 45,96\% e correlacionou-se com a temperatura e a demanda evaporativa da atmosfera. Genótipos com maiores tempos térmicos entre o período de máxima emissão de flores e o final da floração geralmente apresentam maiores percentagens de abortamento de flores. O ajuste dos dados de emissão de flores aos de soma térmica do período da floração, por meio de modelo logístico, permite simular a dinâmica floral de híbridos de canola e mostarda castanha.

Termos para indexação: Brassica juncea, Brassica napus, deiscência natural, soma térmica, umidade relativa.

\section{Floral dynamics and flower abortion in hybrids of canola and Indian mustard}

\begin{abstract}
The objective of this work was to evaluate the floral dynamics and to determine the index of flower abortion in canola (Brassica napus) and Indian mustard (Brassica juncea) hybrids, as well as to determine their relation with meteorological conditions of southern Brazil. During flowering, ten hybrids of canola and two of Indian mustard were evaluated every three days as to the number of open flowers, pods, and aborted flowers. The cumulative and the relative number of flowers were used to evaluate floral dynamics. The relation of these numbers with the accumulated thermal sum during flowering was determined with a logistic model. Groups of genotypes with different flowering rates were identified using the model coefficients. Flower abortion among hybrids ranged from 10.53 to $45.96 \%$ and was correlated with temperature and hydric demand of the atmosphere. Genotypes with larger thermal times between the peak and the end of flowering generally had higher percentages of flower abortion. The adjustment of data from flower emission to those of the thermal sum of the flowering period, using a logistic model, allows simulating floral dynamics of hybrids of canola and Indian mustard.
\end{abstract}

Index terms: Brassica juncea, Brassica napus, natural dehiscence, thermal sum, relative humidity.

\section{Introdução}

A cultura da canola (Brassica napus L.) foi introduzida no Rio Grande do Sul em meados da década de 1970 e vem ganhando destaque como alternativa produtiva rentável para a estação fria, nos estados do Sul do Brasil e, também, em outras regiões do país. A adaptação climática da espécie está entre as principais limitações para manter a expansão de seu cultivo, especialmente no que se refere ao efeito térmico sobre o pegamento de flores (Morrison \& Stewart, 2002) e a deiscência natural de grãos (Thomas, 2003). A mostarda castanha [Brassica juncea (L.) Czern.] é um parente próximo da canola que tem sido melhorado como alternativa a essa oleaginosa, para regiões mais quentes e secas (McCaffery et al., 2009).

A avaliação do efeito da temperatura sobre a floração da canola é importante, uma vez que os estudos de desempenho de híbridos e o estabelecimento de novas áreas de produção comercial no Brasil extrapolam 
as áreas de clima subtropical (Tomm et al., 2004). Além disso, o cenário de aquecimento global pode comprometer o estabelecimento e a manutenção de áreas produtivas em baixas latitudes (Young, 2004). Morrison (1993), em estudo com câmaras de crescimento, observou a ocorrência de esterilidade e queda de flores em temperaturas iguais ou superiores a $27^{\circ} \mathrm{C}$. Esse limite térmico também foi relatado por outros autores (Morrison \& Stewart, 2002; Young, 2004; Wang et al., 2006).

Outra dificuldade importante para o cultivo da canola é o fato de as síliquas não se desenvolverem e amadurecerem ao mesmo tempo (Marchiori Junior et al., 2002). Assim, a deiscência natural tem sido responsável por perdas de mais de $30 \%$ no rendimento da cultura (Thomas, 2003). A dinâmica floral da planta é diretamente influenciada pela temperatura do ar e está relacionada a perdas por deiscência natural de síliquas maduras, antes da colheita. Portanto, a caracterização da dinâmica floral para variados materiais genéticos pode auxiliar na minimização das perdas.

O objetivo deste trabalho foi avaliar a dinâmica floral e determinar o índice de abortamento de flores de híbridos de canola e mostarda castanha, bem como determinar suas relações com as condições meteorológicas da região Sul do Brasil.

\section{Material e Métodos}

O experimento foi conduzido no campus de Frederico Westphalen, $\mathrm{RS}\left(27^{\circ} 23^{\prime} \mathrm{S}\right.$ e $53^{\circ} 25^{\prime} \mathrm{W}$, a $461 \mathrm{~m}$ de altitude), da Universidade Federal de Santa Maria (UFSM), em uma área de Latossolo Vermelho. O clima da região é do tipo Cfa, conforme a classificação de Köppen. Em 2009, foram avaliados os híbridos de canola Hyola 61, Hyola 401, Hyola 433, Hyola 432, Hyola 411, Hyola 76, H4815, K9209, I4403, K911, e os híbridos de mostarda Q6501 e Q6503. A semeadura foi realizada em 28 de maio. Em 2010, o experimento foi instalado em área vizinha, tendo-se avaliado apenas os híbridos Hyola 61 e Hyola 433, com semeadura em 27 de maio. Nos dois experimentos, o estande final foi de 40 plantas por metro quadrado.

As parcelas experimentais foram formadas por cinco linhas de semeadura, com $5 \mathrm{~m}$ de comprimento, espaçadas em $0,34 \mathrm{~m}$ entre si. $\mathrm{O}$ delineamento experimental, nos dois anos, foi o de blocos ao acaso, com quatro repetições. No momento da semeadura, realizou-se a adubação química de base de acordo com a análise química do solo e com as indicações, para a cultura da canola, do manual de adubação e calagem para os estados do Rio Grande do Sul e Santa Catarina (2004).

Para avaliar a dinâmica floral nos dois anos, foram demarcadas, no período vegetativo, duas plantas da linha central de cultivo, em cada parcela, num total de oito plantas por híbrido. As avaliações foram realizadas três vezes por semana, tendo sido iniciadas quando a parcela apresentava $50 \%$ das plantas com pelo menos uma flor e finalizadas por ocasião da ausência total de flores. Nas avaliações, determinou-se o número de flores abertas, de síliquas (fruto seco deiscente) e de flores abortadas, nas hastes principais e secundárias das plantas. Consideraram-se, como flores abortadas, as que apresentavam apenas o pedúnculo, sem o desenvolvimento da síliqua.

A dinâmica floral foi quantificada a partir da determinação do número acumulado de flores (NAF) e do número relativo de flores (NRF). O NAF refere-se ao número total de flores emitidas pela planta até um dado momento, ou seja, o total acumulado de flores, síliquas e flores abortadas registradas até o momento da avaliação. O NRF refere-se ao número total de flores emitidas pela planta até um dado momento, em relação ao total de flores emitidas durante todo o período de avaliação.

Dados de NAF e NRF, obtidos em 2009, foram relacionados às somas térmicas acumuladas nos subperíodos de floração $\left(\mathrm{GD}_{\mathrm{Flor}}\right)$ e vegetativo $\left(\mathrm{GD}_{\mathrm{Veg}}\right)$, e com a soma térmica de todo o ciclo ( $\left.\mathrm{GD}_{\text {Ciclo }}\right)$. Esse procedimento possibilitou o ajuste de modelos para a estimativa da dinâmica floral dos híbridos estudados. Para o cálculo da soma térmica, utilizou-se a equação $\mathrm{GD}=\left(\mathrm{T}_{\text {média }}-\mathrm{T}_{\text {basal }}\right) \times \mathrm{n}$, em que $\mathrm{T}_{\text {média }}$ é a temperatura média diária do ar $\left({ }^{\circ} \mathrm{C}\right) ; \mathrm{T}_{\text {basal }}$ é a temperatura basal inferior, igual a $5^{\circ} \mathrm{C}$ (Morrison et al., 1989); e n é o número de dias do subperíodo ou do período avaliado.

Para normalizar a soma térmica acumulada no subperíodo floração ( $\mathrm{GD}_{\text {Flor.Nor. }}$ ), utilizou-se a escala percentual de 0 (início da floração) a 100\% (em graus-dia acumulados ao final do subperíodo floração), ou, em escala decimal, de 0 a 1.

Realizou-se uma pré-análise dos resultados de Del Ponte et al. (2004) e Bolis et al. (2009), que ajustaram

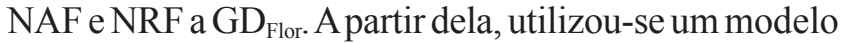
logístico, ajustado a todos os híbridos, conforme a equação: $\mathrm{NAF}$ ou $\mathrm{NRF}=\mathrm{a} /\left[1+\mathrm{e}^{(\mathrm{GDfllor}-\mathrm{Xo}) / b}\right]$, em que as variáveis a, b e Xo são os parâmetros ajustados do 
modelo. O parâmetro Xo foi definido como ponto de máxima emissão de flores. Para diferenciação dos híbridos em grupos homogêneos de floração, foram utilizados os parâmetros Xo e a soma térmica durante o período de floração $\left(\mathrm{GD}_{\text {Flor }}\right)$. A partir do valor de Xo, os híbridos foram divididos em dois grupos, com valor acima ou abaixo do Xo médio. Nos grupos formados, os indivíduos foram divididos com base nas diferenças entre suas somas térmicas, obtidas da máxima emissão de flores (Xo) ao final da floração, tendo-se separado os híbridos em acima ou abaixo do valor médio dessa diferença. No total, foram formados quatro grupos homogêneos de floração.

A significância da regressão entre as variáveis NAF, NRF e GD Flor foi avaliada pelo teste F. Para avaliar os modelos matemáticos empíricos ajustados às curvas de NAF e NRF em função da soma térmica (GD Flor ), foram utilizados o coeficiente de determinação $\left(\mathrm{R}^{2}\right)$, o erro-padrão (EP) da estimativa e o valor $\mathrm{p}$ de significância. Os modelos empíricos de NRF foram validados com o banco de dados de avaliação floral de 2010, tendo-se avaliado os resultados a partir do $\mathrm{R}^{2}$ e da significância da regressão linear entre os dados estimados e os observados.

Resultados das percentagens de abortamento floral para cada híbrido, registrados em 2009, foram submetidos à análise de variância a 5\% de probabilidade e, no caso de diferenças significativas, eles foram analisados pelo teste complementar de Duncan, a $5 \%$ de probabilidade. Os dados de abortamento floral também foram relacionados às unidades de estresse de calor (UEC) e de deficit de pressão de vapor do ar (DPVe), ambos acumulados no subperíodo floração, para os horários com temperatura máxima do ar $\left(\mathrm{T}_{\text {hora }}\right)$ acima dos $27^{\circ} \mathrm{C}$. Os ajustes obtidos foram avaliados a partir do coeficiente de correlação de Pearson, do erro-padrão de estimativa e do nível de significância obtido na análise de variância.

Para o cálculo das UEC, foi adotado o limite térmico (LT) de $27^{\circ} \mathrm{C}$, tendo-se utilizado a equação: $\mathrm{UEC}=\sum\left(\mathrm{T}_{\text {hora }}-\mathrm{LT}\right)$, em que $\sum$ representa o somatório térmico horário, no subperíodo floração, das diferenças positivas de temperatura para ( $\left.\mathrm{T}_{\text {hora }}-\mathrm{LT}\right)$.

A variável deficit de pressão de vapor no ar (DPVe), para horários com temperatura do ar superior a $27^{\circ} \mathrm{C}$, foi calculada a partir da equação $\mathrm{DPVe}=\sum$ es - ea, em que es é a pressão de saturação de vapor d'água do ar $(\mathrm{kPa})$, e ea é a pressão parcial de vapor d'água do ar
$(\mathrm{kPa})$, determinadas, respectivamente, pelas equações: es $=0,6108 \times 10^{7,5 \text { Thora } /(237,3+\text { Thora })}$ e ea $=$ es $\times$ UR/100, em que UR é a umidade relativa do ar (\%).

Os dados meteorológicos, medidos e armazenados em escala horária, foram obtidos junto à estação meteorológica automática do Instituto Nacional de Meteorologia (Inmet), distante $200 \mathrm{~m}$ dos experimentos. Para avaliar a umidade do solo ao longo do ciclo da cultura, utilizou-se a evapotranspiração relativa $(\mathrm{ETr} / \mathrm{ETc})$, referente à fração entre a evapotranspiração real (ETr) e a evapotranspiração máxima da cultura (ETc). A ETr foi determinada a partir de balanço hídrico diário da cultura (Thornthwaite \& Mather, 1955), tendo-se adotado $50 \mathrm{~mm}$ como a capacidade de armazenamento de água no solo, valor esse arbitrário, que varia com o tipo de solo. Para o cálculo daETc, foram multiplicados a evapotranspiração de referência (ETo) (Penman-Monteith) e o coeficiente de cultura (Kc), adaptado de Allen et al. (1998), com valores de $0,35,0,55$ e 0,75 para o primeiro, o segundo e o terceiro terço do período vegetativo; de 1,15 para floração; e de 0,35 para maturação.

\section{Resultados e Discussão}

Nos subperíodos de floração, tanto em 2009 quanto em 2010, houve registros de temperaturas máximas do ar (Tmax) superiores à $27^{\circ} \mathrm{C}$, considerada temperatura limite, a partir da qual há ocorrência de abortamento floral (Morrison, 1993). Em 32\% dos dias do subperíodo de floração, em 2009, a Tmax excedeu os $27^{\circ} \mathrm{C}$, o que acumulou, na média dos 12 híbridos avaliados, $84^{\circ} \mathrm{C}$ de UEC, por dia. Para o mesmo subperíodo de desenvolvimento, em 2010, foram observados menos dias com Tmax acima dos $27^{\circ} \mathrm{C}$, tendo-se acumulado, em média, $64^{\circ} \mathrm{C}$ de UEC por dia. Valores negativos de temperatura do ar, também prejudiciais à floração (Thomas, 2003; Dalmago et al., 2010), não foram registrados na estação meteorológica, durante a floração da canola. Entretanto, em 2010, entre o sétimo e o oitavo decêndio do ciclo da cultura, correspondente a sua plena floração, houve registro uma temperatura muito baixa $\left(0,6^{\circ} \mathrm{C}\right)$, que remete a uma provável temperatura negativa no nível do dossel. A temperatura média diária oscilou entre 10 e $20^{\circ} \mathrm{C}$, em ambos os anos de experimento, e estas temperaturas foram favoráveis ao desenvolvimento da cultura (Thomas, 2003).

A partir da evapotranspiração relativa (ETr/ETc), foi possível determinar a ocorrência de deficiência 
hídrica e o tempo a que os híbridos foram expostos a esta, nos experimentos de 2009 e 2010 . Apesar dos elevados valores de precipitação acumulados no ciclo da canola em 2009 (821 mm) e em 2010 (631 mm), relatados como suficientes para o seu cultivo (Thomas, 2003; Tesfamariam et al., 2010), foram observados valores de deficiência hídrica relativa acumulada de $15 \%(E T r=181 \mathrm{~mm}, E T c=214 \mathrm{~mm})$ e $27 \%$ $(\mathrm{ETr}=147 \mathrm{~mm}, \mathrm{ETc}=202 \mathrm{~mm})$, durante a floração em 2009 e 2010, respectivamente. Valores mínimos de $\mathrm{ETr} / \mathrm{ETc}$ superaram 0,6, em 2009, e 0,5, em 2010, por 5 e 15 dias, durante a floração, respectivamente. Conforme Thomas (2003), tanto a temperatura elevada como a deficiência hídrica acentuam o abortamento floral em canola. Os dados acumulados de radiação solar global, para o ciclo da canola, foram de $1.807 \mathrm{MJ} \mathrm{m}^{-2}$, em 2009, e de $1.769 \mathrm{MJ} \mathrm{m}^{-2}$, em 2010.

O início da floração dos híbridos ocorreu com somas térmicas de 407 a $720^{\circ} \mathrm{C}$-dia (Tabela 1). O híbrido Q6501 foi o mais precoce, enquanto os híbridos I4403, K911 e Hyola 76 foram os mais tardios, em 2009, e o Hyola 61, em 2010. Esses híbridos tardios retardaram em mais de $200^{\circ} \mathrm{C}$-dia o início da floração, quando comparados aos mais precoces. Esse comportamento também foi observado com o final da floração, que foi atingida com $294^{\circ} \mathrm{C}$-dia, no híbrido Q6503, e com $446^{\circ} \mathrm{C}$-dia, no Hyola 433. O híbrido Q6501 foi o que apresentou o maior número acumulado de flores, que diferiu significativamente do apresentado pelos híbridos Q6503, Hyola 61 e Hyola 411, em 2009. Em 2010, os híbridos Hyola 61 e Hyola 433 não apresentaram diferenças quanto ao número total de flores emitidas, como no ano anterior.

Ajustes efetuados nas análises de regressão

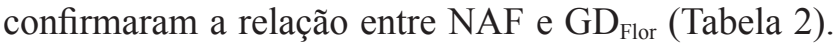
De 56 a $92 \%$ da variação em NAF foi explicada $\left(R^{2}\right)$ pela variação em $\mathrm{GD}_{\text {Flor }}$, por meio do modelo proposto. Ainda conforme avaliação estatística, o erro-padrão de até 109,75 , para estimativa de NAF do híbrido I4403, relativo à média de 408,03 flores por planta, pode ser considerado expressivo e pode estar associado à variabilidade entre plantas do mesmo híbrido.

Quanto ao abortamento floral, a emissão de um maior número de flores por planta (Tabelas 1 e 2) é uma característica desejável, principalmente quando o subperíodo floração é demasiadamente longo e aumenta a probabilidade de ocorrência de eventos adversos. Nas condições climáticas do Rio Grande do Sul, no entanto, as perdas produtivas em canola estão muito menos associadas a esses eventos do que à desuniformidade de maturação, que resulta na deiscência das síliquas secas enquanto ainda há síliquas verdes e, até mesmo, flores no ápice da planta (Thomas, 2003; Tomm, 2007; Marchiori Junior et al., 2002; Dalmago et al., 2008). Isso caracteriza padrão de desenvolvimento indeterminado (Gan et al., 2004;

Tabela 1. Datas de início e fim de floração, tempo de floração (dias), número acumulado total de flores (NTF) e soma térmica acumulada no ciclo $\left(\mathrm{GD}_{\text {Ciclo }}\right)$, no subperíodo vegetativo $\left(\mathrm{GD}_{\mathrm{Veg}}\right)$ e no subperíodo floração $\left(\mathrm{GD}_{\text {Flor }}\right)$ de híbridos de canola e $\operatorname{mostarda} a^{(1)}$.

\begin{tabular}{|c|c|c|c|c|c|c|c|}
\hline \multirow[t]{2}{*}{ Híbrido } & \multicolumn{2}{|c|}{ Floração } & \multirow[t]{2}{*}{ Dias } & \multirow[t]{2}{*}{ NTF } & \multicolumn{3}{|c|}{ Soma térmica $\left({ }^{\circ} \mathrm{C}\right.$-dia $)$} \\
\hline & Início & Fim & & & $\mathrm{GD}_{\text {Ciclo }}$ & $\mathrm{GD}_{\mathrm{Veg}}$ & GD $_{\text {Flor }}$ \\
\hline Q6501 & $31 / 7 / 2009$ & $31 / 8 / 2009$ & 32 & $428 \mathrm{a}$ & 1.138 & 407 & 372 \\
\hline I4403 & $18 / 8 / 2009$ & $17 / 9 / 2009$ & 31 & $392 \mathrm{ab}$ & 1.371 & 617 & 365 \\
\hline K911 & $18 / 8 / 2009$ & $19 / 9 / 2009$ & 33 & $314 \mathrm{ab}$ & 1.464 & 617 & 387 \\
\hline Hyola 401 & $7 / 8 / 2009$ & 4/9/2009 & 29 & 291ab & 1.236 & 479 & 393 \\
\hline Hyola 433 & $10 / 8 / 2009$ & 2/9/2009 & 24 & $283 \mathrm{ab}$ & 1.223 & 516 & 297 \\
\hline Hyola 76 & $18 / 8 / 2009$ & $17 / 9 / 2009$ & 31 & $281 \mathrm{ab}$ & 1.306 & 617 & 365 \\
\hline K9209 & $12 / 8 / 2009$ & $10 / 9 / 2009$ & 33 & $273 \mathrm{ab}$ & 1.265 & 528 & 371 \\
\hline $\mathrm{H} 4815$ & $4 / 8 / 2009$ & 2/9/2009 & 30 & $251 \mathrm{ab}$ & 1.197 & 441 & 405 \\
\hline Hyola 432 & $10 / 8 / 2009$ & $7 / 9 / 2009$ & 29 & $240 \mathrm{ab}$ & 1.250 & 516 & 364 \\
\hline Q6503 & $7 / 8 / 2009$ & $31 / 8 / 2009$ & 25 & $208 b$ & 1.197 & 479 & 294 \\
\hline Hyola 61 & $14 / 8 / 2009$ & $10 / 9 / 2009$ & 28 & $207 \mathrm{~b}$ & 1.306 & 552 & 340 \\
\hline Hyola 411 & 7/8/2009 & 4/9/2009 & 29 & $205 b$ & 1.236 & 479 & 352 \\
\hline Hyola 61 & $23 / 8 / 2010$ & $15 / 9 / 2010$ & 38 & $352 \mathrm{a}$ & 1.261 & 720 & 352 \\
\hline Hyola 433 & 9/8/2010 & $11 / 9 / 2010$ & 34 & $281 \mathrm{a}$ & 1.261 & 576 & 446 \\
\hline
\end{tabular}

(1) Médias de NTF seguidas de letras iguais não diferem pelo teste de Tukey, a 5\% de probabilidade. Na análise individual das médias para 2009, o coeficiente de variação foi de $37 \%$, e, em 2010 , o coeficiente de variação foi de $43 \%$. 
Koenig et al., 2011), que tem, como vantagem, uma maior capacidade de recuperação das plantas a eventos de estresse e confere plasticidade fenotípica à cultura (Gan et al., 2004).

Auniformidade da maturação das síliquas fertilizadas relaciona-se, também, ao tempo do subperíodo de floração e ao número de flores produzidas (Tabela 1) (Morrison \& Stewart, 2002). Dessa forma, de acordo com o ponto de inflexão das curvas (Xo) (Tabela 2), que representa a soma térmica para a máxima taxa de emissão de flores, o híbrido Q6503 destacou-se pela precocidade e o Hyola 401 pela emissão tardia de flores, com $78^{\circ} \mathrm{C}$-dia de diferença entre seus pontos de máxima emissão de flores. Porém, ao se avaliar o tempo térmico transcorrido entre Xo (Tabela 2) e o fim da floração (Tabela 1), sobressaiu-se o híbrido Hyola 433, cuja inflexão da curva ocorreu com $122,31^{\circ} \mathrm{C}$-dia. Assim, restaram, pelo menos, $17^{\circ} \mathrm{C}$-dia até o final da floração, com um total de flores produzidas superior à média de todos os híbridos. Essas mesmas características também foram observadas para Hyola 411 e Q6501. Apesar de o híbrido Hyola 76 produzir um total de flores superior à média dos demais híbridos, ele apresentou $\mathrm{GD}_{\text {Flor }}$ superior à média, com maior intervalo de tempo térmico $\left(243^{\circ} \mathrm{C}\right.$-dia $)$ entre Xo e o final do subperíodo floração.

A partir dos resultados de NAF e GD $_{\text {Flor, }}$ e ao se tomar, como referência, os valores médios de Xo e da diferença entre Xo e GD Flor (Tabelas 1 e 2), para expressar os resultados da relação entre NRF e

Tabela 2. Coeficientes a e b do modelo matemático logístico e parâmetro Xo (ponto de máxima emissão de flores) das análises de regressão entre o número acumulado de flores e a soma térmica acumulada durante a floração ${ }^{(1)}$.

\begin{tabular}{lcccccc}
\hline Híbrido & $\mathrm{a}$ & $\mathrm{b}$ & $\mathrm{Xo}$ & $\mathrm{R}^{2}$ & $\mathrm{EP}$ & $\mathrm{p}$ \\
\hline Hyola 401 & 297,81 & 52,29 & 180,18 & 0,92 & 31,22 & $<0,0001$ \\
H4815 & 255,71 & 50,76 & 166,80 & 0,60 & 72,89 & $<0,0001$ \\
Q6501 & 427,36 & 37,90 & 174,37 & 0,77 & 95,46 & $<0,0001$ \\
Hyola 411 & 207,11 & 41,45 & 135,85 & 0,81 & 38,88 & $<0,0001$ \\
Q6503 & 210,23 & 37,37 & 102,23 & 0,83 & 36,17 & $<0,0001$ \\
Hyola 432 & 247,90 & 56,57 & 132,44 & 0,82 & 39,42 & $<0,0001$ \\
K9209 & 277,74 & 61,51 & 163,46 & 0,72 & 57,52 & $<0,0001$ \\
Hyola 433 & 288,14 & 45,59 & 122,31 & 0,77 & 55,39 & $<0,0001$ \\
Hyola 61 & 210,92 & 52,11 & 109,64 & 0,56 & 57,83 & $<0,0001$ \\
Hyola 76 & 289,45 & 65,65 & 122,09 & 0,78 & 49,14 & $<0,0001$ \\
K911 & 323,85 & 69,75 & 163,46 & 0,90 & 35,85 & $<0,0001$ \\
I4403 & 408,03 & 58,98 & 125,99 & 0,61 & 109,75 & $<0,0001$ \\
Média & 294,30 & 56,38 & 148,32 & 0,62 & 75,81 & $<0,0001$ \\
\hline
\end{tabular}

${ }^{(1)} \mathrm{EP}$, erro-padrão da estimativa; $\mathrm{p}$, significância estatística.
$\mathrm{GD}_{\text {Flor.Nor. }}$ (Figura 1), foram geradas equações para cada agrupamento de híbridos quanto à precocidade/ atraso para atingir a máxima taxa de emissão de flores (Xo) e quanto à diferença entre Xo e GD Flor. Dessa forma, ficaram, no agrupamento 1, os híbridos Hyola 401, Q6501 e K9209; no agrupamento 2, os híbridos Hyola 411, Q6503 e Hyola 433; no agrupamento 3, os híbridos H4815 e K911; e, no agrupamento 4, os híbridos Hyola 432, Hyola 61, Hyola 76 e I4403.

Com base nos coeficientes gerados (Figura 1), foi possível verificar que o agrupamento 1 apresentou maior uniformidade de floração em comparação aos demais, pois o tempo entre Xo e o final da floração foi menor. Além disso, o valor de $b$, que indica o tempo de início da taxa de emissão linear da equação logística, também foi menor, ou seja, a floração ocorreu de forma mais concentrada. Os agrupamentos 2 e 3 apresentaram resultados intermediários, em que o coeficiente Xo é maior no grupo 3 e o coeficiente b é menor no grupo 2. Quanto ao agrupamento 4, com base nos resultados do ajuste logístico, houve tendência de floração mais desuniforme em comparação aos demais, já que o parâmetro Xo apresentou os menores valores, e a variável $b$, os maiores, o que indica que, neste agrupamento, os híbridos ficam mais tempo floridos e apresentam baixa taxa de emissão de flores.

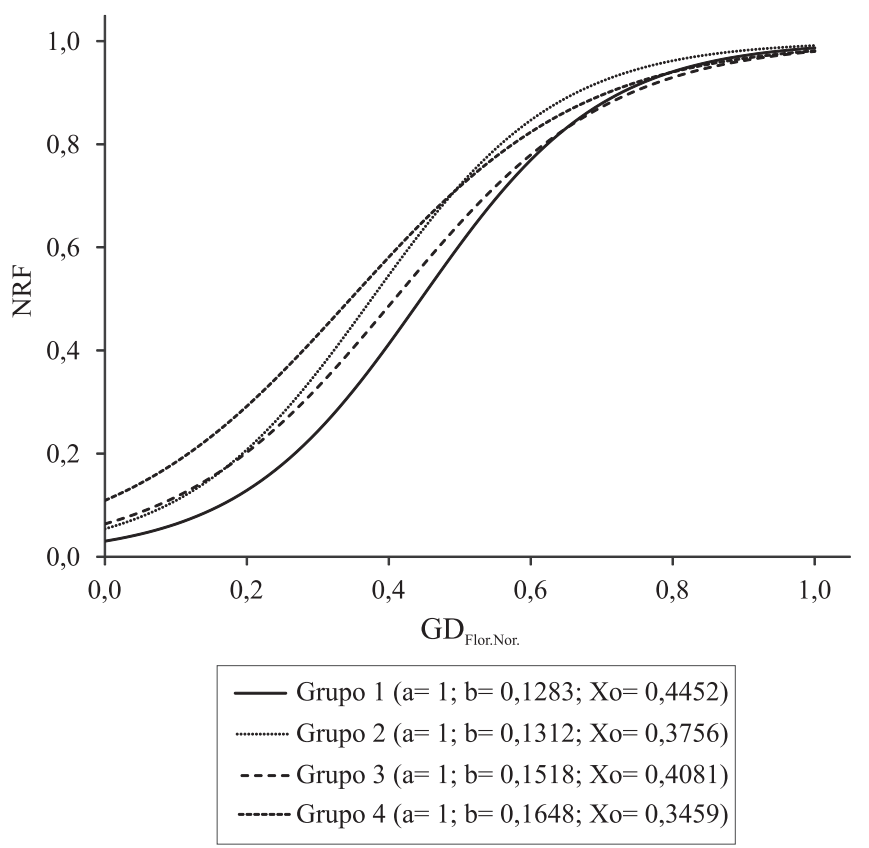

Figura 1. Relações entre número relativo de flores (NRF) e soma térmica normalizada na floração $\left(\mathrm{GD}_{\text {Flor.Nor. }}\right)$ para os agrupamentos de híbridos de canola. 
A menor deiscência de síliquas, em razão do florescimento mais uniforme, e as maiores taxas de emissão de flores indicam que os híbridos do grupo 1 (Figura 1) são materiais mais indicados para enfrentar períodos de condições meteorológicas adversas, seguidos dos grupos 2, 3 e 4 . Ao se adotar o critério de uniformidade de floração e o de taxa de emissão de flores, o híbrido preferencial seria o Q6501. Cabe ressaltar, entretanto, que, no presente trabalho, não se verificou relação clara entre produtividade, grupo de floração e taxa de emissão total de flores, apesar de ser comum, na literatura, o relato de diminuição na produtividade pelo aumento do abortamento de flores (Gan et al., 2004).

A validação dos coeficientes obtidos para os agrupamentos foi feita por meio de dados independentes, obtidos em 2010 para os híbridos Hyola 61 e Hyola 433, pertencentes aos grupos 4 e 2, respectivamente(Figura 2). Para o Hyola 61, constatouse que os valores estimados e os observados de NRF estavam próximos, com coeficiente de inclinação da equação linear entre esses valores de 0,93 , ou seja, o modelo de estimativa estaria superestimando em $7 \%$ os valores em relação aos observados. No caso do híbrido Hyola 433, essa superestimativa aumentou para 17\%. Esses resultados podem ser atribuídos às diferenças térmicas entre os experimentos de calibração (2009) e de validação (2010), pois, no início da floração em
2010, ocorreram valores de temperatura do ar menores do que em 2009. Conforme os dados meteorológicos dos dois anos experimentais, foram registradas, em 2009 , temperaturas do ar superiores a $27^{\circ} \mathrm{C}$ no decorrer do subperíodo floração, condição apontada como grande responsável pelo abortamento floral em canola (Morrison, 1993; Angadi et al., 2000). A percentagem de flores abortadas por planta, nos 12 híbridos testados, variou de 10,53\% (Hyola 411) a $45,96 \%$ (Hyola 76 ), valores estatisticamente distintos (Tabela 3).

Os quatro híbridos com menor percentagem de abortamento floral (Hyola 411, Q6501, Q6503 e K9209) apresentaram menor tempo térmico entre Xo e o fim da floração do que a média dos 12 híbridos (Tabela 2). Já os respectivos tempos térmicos dos três híbridos com maior abortamento (Hyola 76, K911 e I4403) foram superiores à média dos 12 híbridos (Tabelas 1 e 2). De acordo com Faraji et al. (2008), a produção de flores em canola é duas vezes maior que o número final de síliquas, e os diferentes percentuais de abortamento floral não devem ser atribuídos direta e exclusivamente ao fator genético, uma vez que, por apresentarem diferentes dinâmicas florais, no que se refere ao tempo total do subperíodo floração (Tabelas 1 e 2, Figura 1), cada híbrido acaba exposto a diferentes adversidades; no caso específico do presente trabalho, ao excesso térmico.
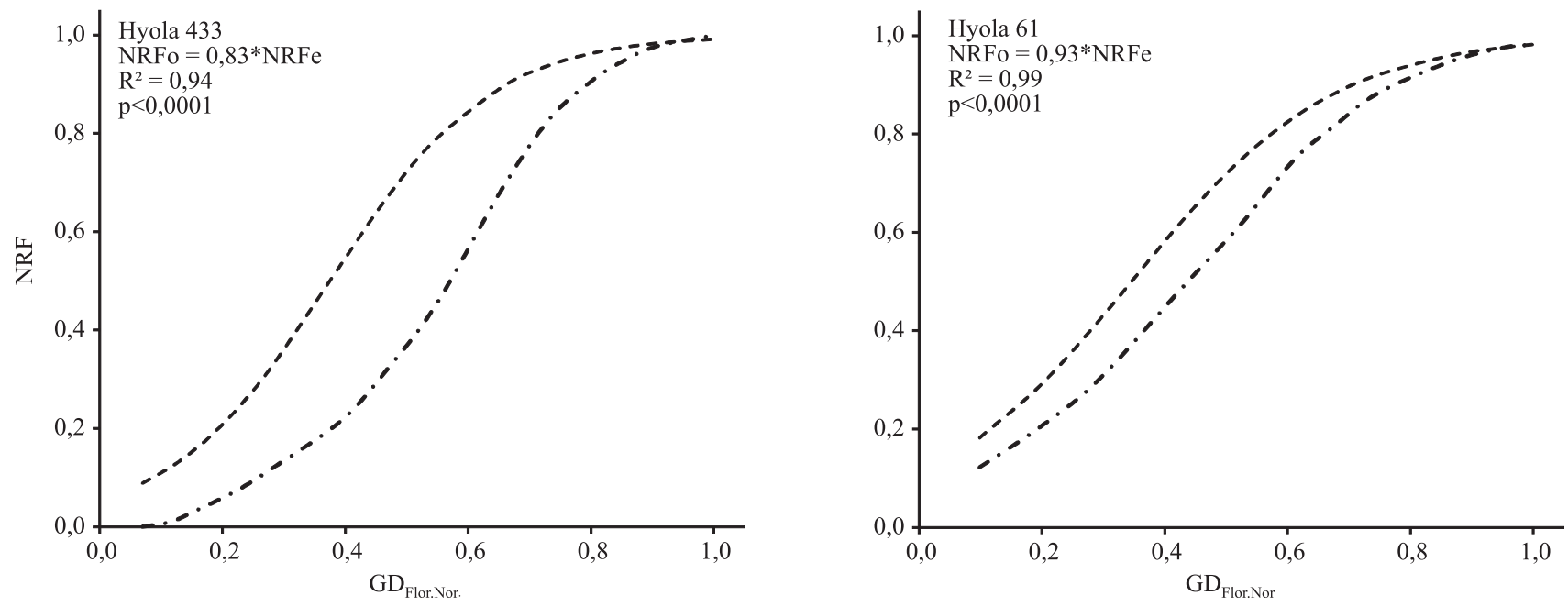

$-\cdot-\cdot$ Observado (o) - - - - Estimado (e)

Figura 2. Validação dos coeficientes estimados para os grupos 2 (Hyola 433) e 4 (Hyola 61), para número relativo de flores (NRF), em função de soma térmica normalizada $\left(G_{\text {Flor.Nor. }}\right)$, com dados independentes obtidos em 2010. 
Tabela 3. Percentual total de flores abortadas (AB), coeficiente de correlação de Pearson (r) e erro-padrão da estimativa (EP) para as relações entre percentual de abortamento de flores, unidades de estresse de calor (UEC) e deficit de pressão de vapor de estresse (DPVe), para híbridos de canola e de mostarda ${ }^{(1)}$.

\begin{tabular}{|c|c|c|c|c|c|c|c|}
\hline \multirow[t]{2}{*}{ Híbrido } & \multirow{2}{*}{$\begin{array}{l}\text { AB } \\
(\%)\end{array}$} & \multicolumn{2}{|c|}{ AB x UEC } & \multicolumn{2}{|c|}{$\mathrm{AB} \times \mathrm{DPVe}$} & \multicolumn{2}{|c|}{$\mathrm{AB} \times(\mathrm{UEC}+\mathrm{DPVe})$} \\
\hline & & $\mathrm{r}$ & EP & $\mathrm{r}$ & $\mathrm{EP}$ & $\mathrm{r}$ & EP \\
\hline Hyola 401 & $26,36 \mathrm{bcd}$ & $0,95 * *$ & 3,35 & $0,94 * *$ & 3,78 & $0,95 * *$ & 3,53 \\
\hline $\mathrm{H} 4815$ & $22,02 \mathrm{abc}$ & $0,80 * *$ & 6,19 & $0,85^{* *}$ & 5,36 & $0,89 * *$ & 4,97 \\
\hline Q6501 & $16,64 \mathrm{ab}$ & $0,80 * *$ & 4,69 & $0,83 * *$ & 4,39 & $0,85 * *$ & 4,33 \\
\hline Hyola 411 & $10,53 \mathrm{a}$ & $0,97 * *$ & 1,14 & $0,97 * *$ & 0,98 & $0,97 * *$ & 1,03 \\
\hline Q6503 & $18,33 \mathrm{ab}$ & $0,76^{* *}$ & 5,62 & $0,79 * *$ & 5,30 & $0,82 * *$ & 5,32 \\
\hline Hyola 432 & $26,49 \mathrm{bcd}$ & $0,97 * *$ & 2,84 & $0,95 * *$ & 3,86 & $0,98 * *$ & 2,75 \\
\hline K9209 & $21,64 \mathrm{ab}$ & $0,92 * *$ & 3,26 & $0,88 * *$ & 4,08 & $0,96^{* *}$ & 2,45 \\
\hline Hyola 433 & $27,26 \mathrm{bcd}$ & $0,95 * *$ & 3,36 & $0,94 * *$ & 3,56 & $0,95^{* *}$ & 3,56 \\
\hline Hyola 61 & $24,37 \mathrm{bcd}$ & $0,99 * *$ & 1,83 & $0,97 * *$ & 2,87 & $0,99 * *$ & 1,43 \\
\hline Hyola 76 & $45,96 \mathrm{e}$ & $0,92 * *$ & 8,66 & $0,89 * *$ & 9,86 & $0,95^{* *}$ & 6,91 \\
\hline K911 & $44,93 \mathrm{de}$ & $0,95^{* *}$ & 6,03 & $0,92 * *$ & 7,12 & $0,97 * *$ & 4,53 \\
\hline I4403 & 34,14 cde & $0,94 * *$ & 5,54 & $0,92 * *$ & 6,30 & $0,95^{* *}$ & 4,99 \\
\hline
\end{tabular}

${ }^{(1)}$ Médias seguidas de letras iguais não diferem pelo teste de Ducan, a $5 \%$ de probabilidade. ${ }^{* *}$ Significativo a $1 \%$ de probabilidade.

Faragi (2010) observou que a relação síliquas/flores é influenciada de forma quadrática pelo índice de área foliar e pela quantidade de matéria seca produzida pela parte aérea, e de forma linear positiva pelo tempo da duração da área foliar. O autor relata que, quando as condições ambientais são desfavoráveis para o desenvolvimento da planta, a relação síliquas/flores tende a diminuir, o que aumenta a taxa de abortamento floral.

Com base no percentual de abortamento floral de cada híbrido (Tabela 3), para as unidades de estresse de calor (UEC) e o deficit de pressão de vapor (DPVe), e nos índices estatísticos ( $r>0,76$ e EP $<10)$, observouse que o uso de UEC e DPVe, de forma separada ou conjunta, pode auxiliar na geração de modelos de estimativa do percentual de abortamento floral para as culturas de canola e mostarda (Tabela 3). Esse fato foi confirmado por Morrison \& Stewart (2002), que relataram correlação negativa entre UEC e a produção de grãos, com o uso da temperatura limite de $29,5^{\circ} \mathrm{C}$. No presente trabalho, ao se utilizar a temperatura do ar limite para a ocorrência de abortamento floral de $27^{\circ} \mathrm{C}$, constatou-se que a correlação entre essas variáveis variou entre 0,76 e 0,99 , com diferentes níveis de erro-padrão (EP), o que indica que os híbridos respondem de modo diferente a essas temperaturas e podem apresentar diferentes limiares de temperatura máxima do ar. Embora Morrison (1993) tenha verificado a ocorrência de abortamento em temperaturas do ar superiores a $27^{\circ} \mathrm{C}$, Morrison \& Stewart (2002) encontraram valores limiares de temperatura entre 29,3 e $29,9^{\circ} \mathrm{C}$. A conjugação das variáveis UEC e DPVe possibilitou a obtenção de maiores correlações com o abortamento floral (Tabela 3), principalmente em relação aos híbridos
Q6503, H4815 e Q6501, que apresentaram pior desempenho quando essas variáveis foram utilizadas de forma independente.

Observou-se menor eficiência dos modelos para os híbridos de mostarda, indício de que características específicas da espécie devem estar envolvidas na resposta desta aos parâmetros avaliados. Segundo Angadi et al. (2000), cultivares de mostarda são mais bem adaptadas a condições de semiárido e, consequentemente, a excessos térmicos. De acordo com Gan et al. (2004), a mostarda consegue explorar melhor a produção de flores nos ramos laterais após um período de estresse ambiental em comparação a outras espécies de Brassica. Esses resultados ajudam a explicar a menor eficiência do modelo para os híbridos de mostarda, pois este foi desenvolvido com base em critérios de abortamento floral para B. napus.

\section{Conclusões}

1. O ajuste dos dados de emissão de flores aos de soma térmica do período da floração, por meio de modelo logístico, permite simular a dinâmica floral de híbridos de canola e mostarda.

2. Os coeficientes desse modelo permitem distinguir grupos de genótipos quanto à taxa de emissão floral.

3. Maiores tempos térmicos entre o período de máxima emissão de flores e o final da floração estão normalmente associados a maiores percentagens de abortamento floral.

4. A condição térmica e a demanda evaporativa da atmosfera correlacionam-se com o abortamento floral, e essas variáveis devem ser incluídas em modelos para estimação do abortamento. 


\section{Agradecimentos}

À Embrapa Trigo, pela disponibilização das sementes de canola e mostarda para a realização deste experimento.

\section{Referências}

ALLEN, R.G.; PEREIRA, L.S.; RAES, D.; SMITH, M. Crop evapotranspiration: guidelines for computing crop water requirements. Roma: FAO, 1998. 300p. (FAO. Irrigation and drainage paper, 56).

ANGADI, S.V.; CUTFORTH, H.W.; MILLER, P.R.; MCCONKEY, B.G.; ENTZ, M.H.; BRANDT, A.; OLKMAR, K.M. Response of three Brassica species to high temperature stress during reproductive growth. Canadian Journal of Plant Science, v.80, p.693-701, 2000. DOI: 10.4141/P99-152.

BOLIS, L.M.; MÜLLER, A.L.; DALMAGO, G.A.; FERNANDES, J.M.C.; SANTI, A.; CUNHA, G.R. da; SCHWEIG, E. Modelagem da floração da canola em resposta a soma térmica. In: CONGRESSO BRASILEIRO DE AGROMETEOROLOGIA, 16., 2009, Belo Horizonte, MG. Mudanças climáticas, recursos hídricos e energia para uma agricultura sustentável: anais. Campinas: SBA: Viçosa: UFV: Sete Lagoas: Embrapa Milho e Sorgo, 2009. 1 CD ROM.

DALMAGO, G.A.; CUNHA, G.R. da; SANTI, A.; PIRES, J.L.F.; MÜLLER, A.L.; BOLIS, L.M. Aclimatação ao frio e dano por geada em canola. Pesquisa Agropecuária Brasileira, v.45, p.933-943, 2010. DOI: 10.1590/S0100-204X2010000900001.

DALMAGO, G.A.; CUNHA, G.R. da; TOMM, G.O.; PIRES, J.L.F.; SANTI, A.; PASINATO, A.; SCHWEIG, E.; MÜLLER, A.L. Zoneamento agroclimático de canola para o Rio Grande do Sul. Passo Fundo: Embrapa Trigo, 2008. 10p. (Embrapa Trigo. Comunicado técnico, 252).

DEL PONTE, E.M.; FERNANDES, J.M.C.; PAVAN, W.; PIEROBOM, C.R. Simulação da dinâmica do florescimento do trigo como base para um modelo de risco de giberela. Revista Brasileira de Agrociência, v.10, p.323-331, 2004.

FARAJI, A. Flower formation and pod/flower ratio in canola (Brassica napus L.) affected by assimilates supply around flowering. International Journal of Plant Production, v.4, p.271-280, 2010.

FARAJI, A.; LATIFI, N.; SOLTANI, A.; RAD, A.H.S. Effect of high temperature stress and supplemental irrigation on flower and pod formation in two canola (Brassica napus L.) cultivars at Mediterranean climate. Asian Journal of Plant Sciences, v.7, p.343-351, 2008. DOI: 10.3923/ajps.2008.343.351.

GAN, Y.; ANGADI, S.V.; CUTFORTH, H.; POTTS, D.; ANGADI, V.V.; MCDONALD, C.L. Canola and mustard response to short periods of temperature and water stress at different developmental stages. Canadian Journal of Plant Science, v.84, p.697-704, 2004. DOI: 10.4141/P03-109.
KOENIG, R.T.; HAMMAC, W.A.; PAN, W.L. Canola growth, development, and fertility. Washington: Washington State University, 2011. 6p.

MANUAL de adubação e de calagem para os estados do Rio Grande do Sul e Santa Catarina. 10.ed. Porto Alegre: Sociedade Brasileira de Ciência do Solo - Núcleo Regional Sul - Comissão de Química e Fertilidade do Solo, 2004. 400p.

MARCHIORI JUNIOR, O.; INOUE, M.H.; BRACCINI, A.L.; OLIVEIRA JUNIOR, R.S.; AVILA, M.R.; LAWDER, M.; CONSTANTIN, J. Qualidade e produtividade de sementes de canola (Brassica napus) após aplicação de dessecantes em pré-colheita. Planta Daninha, v.20, p.253-261, 2002. DOI: 10.1590/S0100-83582002000200012.

MCCAFFERY, D.; BAMBACH, R.; HASKINS, B. Brassica juncea in north-western NSW. $2^{\text {nd }}$ ed. Orange: NSW Department of Primary Industries, 2009. 10p. (Primefact, 786).

MORRISON, M.J. Heat stress during reproduction in summer rape. Canadian Journal of Botany, v.71, p.303-308, 1993. DOI: 10.1139/b93-031.

MORRISON, M.J.; STEWART, D.W. Heat stress during flowering in summer Brassica. Crop Science, v.42, p.797-803, 2002. DOI: 10.2135/cropsci2002.0797.

MORRISON, M.J.; MCVETTY, P.B.E.; SHAYKEWICH, C.F. The determination and verification of a baseline temperature for the growth of westar summer rape. Canadian Journal of Plant Science, v.69, p.455-464, 1989. DOI: 10.4141/cjps89-057.

TESFAMARIAM, E.H.; ANNANDALE，J.G.; STEYN，J.M. Water stress effects on winter canola growth and yield. Agronomy Journal, v.102, p.658-666, 2010. DOI: 10.2134/agronj2008.0043.

THOMAS, P. The growers' manual. Winnipeg: Canola Council of Canada, 2003. Available at: <http://www.canolacouncil.org/ canola_growers_manual.aspx>.Accessed on: 1 May 2012.

THORNTHWAITE, C.W.; MATHER, J.R. The water balance. New Jersey: Drexel Institute of Technology, 1955. 104p. (Publications in climatology).

TOMM, G.O. Canola: alternativa de renda e benefícios para os cultivos seguintes. Revista Plantio Direto, v.15, p.4-8, 2006.

TOMM, G.O. Indicativos tecnológicos para a produção de canola no Rio grande do Sul. Passo Fundo: Embrapa Trigo, 2007. 68p. (Embrapa Trigo. Sistemas de produção, 4).

TOMM, G.O.; SOARES, A.L.S.; MELLO, M.A.B. de; DEPINÉ, D.E.; FIGER, E. Desempenho de genótipos de canola em Goiás, em 2004. Passo Fundo: Embrapa Trigo, 2004. 11p.

WANG, J.; GAN, Y.T.; CLARKE, F.; MCDONALD, C.L. Response of chickpea yield to high temperature stress during reproductive development. Crop Science, v.46, p.2171-2178, 2006. DOI: 10.2135/cropsci2006.02.0092.

YOUNG, L.W. High temperature stress of Brassica napus during flowering reduces micro- and megagametophyte fertility, induces fruit abortion, and disrupts seed production. Journal of Experimental Botany, v.55, p.485-495, 2004. DOI: 10.1093/jxb/erh038.

Recebido em 9 de julho de 2012 e aprovado em 30 de janeiro de 2013 